\title{
Serum Markers in Rheumatoid Arthritis: A Longitudinal Study of Patients Undergoing Infliximab Treatment
}

\author{
Oddgeir Selaas, ${ }^{1}$ Hilde H. Nordal, ${ }^{1,2}$ Anne-Kristine Halse, ${ }^{2}$ Johan G. Brun, \\ Roland Jonsson, ${ }^{1}$ and Karl A. Brokstad ${ }^{1}$ \\ ${ }^{1}$ Broegelmann Research Laboratory, Department of Clinical Science, University of Bergen, 5020 Bergen, Norway \\ ${ }^{2}$ Department of Rheumatology, Haukeland University Hospital, University of Bergen, 5020 Bergen, Norway \\ Correspondence should be addressed to Karl A. Brokstad; karl.brokstad@k2.uib.no
}

Received 18 September 2015; Revised 30 October 2015; Accepted 30 November 2015

Academic Editor: Bruce M. Rothschild

Copyright (C) 2015 Oddgeir Selaas et al. This is an open access article distributed under the Creative Commons Attribution License, which permits unrestricted use, distribution, and reproduction in any medium, provided the original work is properly cited.

\begin{abstract}
Objective. The aim of this study was to investigate the clinical effect and serum markers in a cohort of rheumatoid arthritis patients with moderate to high disease activity, participating in an open clinical phase IV study conducted in Norway between 2001 and 2003 receiving infliximab treatment. Method. A total of 39 patients were studied, with a mean age of 54 years and 12-year disease duration. The analyses were performed using serum from patients at four assessment time points: baseline and 3, 6, and 12 months after starting treatment with infliximab. A wide variety of clinical data was collected and disease activity of 28 joints and Simple Disease Activity Index were calculated. The joint erosion was determined by X-ray imaging and the Sharp/van der Heijde score was determined. Serum analysis included multiplex immunoassays for 12 cytokines, 5 matrix metalloproteases, and 2 VEGFs. Results. The majority of the RA patients in this study had initially moderate to high disease activity and the infliximab treatment reduced the disease activity significantly and also reduced any further joint destruction and improved disease status. Most of the serum levels of cytokines and metalloproteases remained unchanged during the course of the study, and we were unable to detect changes in TNF- $\alpha$ in serum. Serum levels of IL-6 and VEGF-A decreased significantly after initiation of infliximab treatment. Conclusion. The serum levels of IL- 6 and VEGF-A may be promising disease markers as they vary with disease progression. The clinical significance of these findings is yet to be determined and has to be confirmed in future clinical trials before being applied in the clinics.
\end{abstract}

\section{Introduction}

Rheumatoid arthritis (RA) is an autoimmune chronic inflammatory disease characterised by persistent synovitis and progressive erosion of the cartilage and the bone in joints, in addition to the presence of autoantibodies like rheumatoid factor (RF) and anti-citrullinated protein/peptide antibodies (ACPA). Left untreated RA can lead to severe damage of joints and immobility and significantly reduce the quality of life. The prevalence of RA is approximately $0.5-1 \%$ of the population and it affects women 2.5 times more frequently than men [1]. Genetic predisposition and smoking are known risk factors for RA, and diagnosis is based on several criteria from both clinical assessments and laboratory analyses. The combination of CRP and ESR and autoantibodies like RF and ACPA are frequently used in the assessment and diagnosis of RA patients [2-4]. In addition, a variety of clinical data are recorded to support the RA diagnosis, including number of tender and swollen joints, high-resolution X-ray of involved joints, evaluation of disease activity using a visual analogue scale, and utilizing mathematical algorithms to determine the overall disease activity like disease activity score of 28 joints (DAS28 [5]) or Simplified Disease Activity Index (SDAI [6, 7]). Clinical diagnosis of RA is based on classification criteria and guidelines from the American College of Rheumatology (ACR) and the European League Against Rheumatism (EULAR). Collaboration between the two resulted in 2010 in a revised set of the older 1987 criteria $[8,9]$. These new classification criteria focus on patients with no prior RA diagnosis and seek to determine definite RA in the absence of alternative diagnoses.

Treating RA patients has traditionally been carried out using immunosuppressive drugs like corticosteroids, disease-modifying antirheumatic drugs (DMARDs) like 
methotrexate, and nonsteroidal anti-inflammatory drugs (NSAIDs). In the last 10-20 years biological DMARDs have been introduced in the treatment of RA. These consist of a variety of cytokine inhibitors and other immune modulators. Due to the high cost of biological DMARDs, it is very important to identify nonresponders at earliest time point, the development of anti-drug antibodies, and disease progression markers. The aim of this study was to evaluate the effect of infliximab treatment on a cohort of advanced RA patients over a one-year period and to identify serum markers that could have relevance to disease progression, remission, or prognostic value.

\section{Material and Methods}

2.1. Patients and Samples. A total of 39 patients (Tables 1 and 2) defined by classification criteria [9] were selected from a cohort of 76 patients participating in an open clinical phase IV study receiving the TNF- $\alpha$ inhibitor Remicade (infliximab). Patients were included between October 2001 and March 2003 and then followed from baseline and up to 3 years after inclusion [10]. The patients were given $3 \mathrm{mg} / \mathrm{kg}$ infliximab at weeks 0,2 , and 6 and then every 8th week concomitantly with methotrexate (11 mg/week) and daily prednisolone. The treatment and sample collection were carried out at three hospitals in Western Norway: Førde Central Hospital, Haukeland University Hospital, and Haugesund Rheumatism Hospital. Rheumatologist assessed the patients and a range of clinical data was recorded. The assessment time points were at baseline and 3, 6, and 12 months afterwards. From the cohort we selected only patients that provided complete sample sets from the chosen assessments points. Samples were stored immediately after collection at $-80^{\circ} \mathrm{C}$.

2.2. Approvals. This study was approved by the Regional Ethical Committee (REK II 84.01) and the Norwegian Medicines Agency. Informed and signed consent was obtained from all patients.

2.3. Disease Activity Scores. We have used two disease activity scores, namely, Disease Activity Score of 28 joints (DAS28) and Simplified Disease Activity Index (SDAI) [5-7]. These are validated scores that describe the overall disease activity and are calculated from both subjective and objective readings, number of swollen joints from 28 joints (SWJ28), number of tender joints from 28 joints (TEN28), C-reactive protein (CRP), erythrocyte sedimentation rate (ESR), patient's global assessment (PGA, pVAS), and physician's global assessment (MDGA, mdVAS). The DAS28 score (0 to 10) is described as follows: a score below 2.6 indicates that the patient is in remission. A DAS28 score from $>2.6$ to $\leq 3.2$ and from $>3.2$ to $\leq 5.1$ indicates low and moderate disease activity, respectively, and a DAS28 score above is indicative of high disease activity. SDAI, a simplified disease index, is also useful (0-100) when not all clinical data are available, with scores above 26 indicating high disease activity. Scores between 11 to 26 are moderate, from 3.3 to 11 show low disease activity, and below 3.3 are considered in remission.
2.4. Analyses of Cytokines and MMPs and VEGFs Levels in Serum. Kits based on the Luminex bead immunoassay technology were used to detect cytokines, matrix metalloproteases (MMPs), and vascular endothelial growth factors (VEGFs) in serum. In order to assay the 12 cytokines, IL$1 \beta$, IL-2, IL-4, IL-5, IL-6, IL-10, IL-12, IL-17A, IL-18, IFN$\gamma$, TNF- $\alpha$, and TNF- $\beta$, we mixed a ProcartaPlex premade multiplex-kit with single-plex kits (Cat. nos. EPX060-10009901, EPX010-10243, EPX010-10221, EPX010-10215, EPX01012017, EPX010-10267, and EPX010-10202 (Affymetrix, eBioscience, Inc., San Diego, USA)). MMP-1, MMP-7, MMP-8, MMP-12, and MMP-13 were assayed by using ProcartaPlex Human MMP-Panel I 5 plex (EPX050-10015-901, Affymetrix, eBioscience). VEGF-A and VEGF-D were measured using the single-plex assays EPX010-10277 and EPX010-12076 (Affymetrix), respectively. We followed the manufacturers recommended procedure for these assays using a Luminex 100 (Luminex Corp., Austin, TX, USA) and STarStation v.2 software (Applied Cytometry, Dinnington, UK).

2.5. Data Processing. All data were processed and analysed, and all graphs have been prepared in GraphPad Prism 6 (GraphPad Software Inc., La Jolla, USA).

\section{Results}

In this longitudinal study we have monitored clinical and serological data from a cohort of 39 RA patients receiving infliximab treatment over one year.

3.1. Disease Activity Scores (DAS). A majority of the patients were in the high disease activity group before treatment was started with an average DAS28 score of $5.3 \pm 0.1$ (Figure 1(a)). After onset of treatment, the majority of patients moved to low and moderate disease activity, with an average DAS28 score of $4.0 \pm 0.2$ after 12 months $(p<0.0001)$.

Similarly, the SDAI score showed that most patients were in the high disease activity group before treatment (Figure 1(b)) with an average SDAI score of 38.8 \pm 2.0 . During treatment the patients as a group moved to moderate and low disease activity with an average of SDAI score 20.0 \pm 2.1 after 12 months $(p<0.0001)$.

3.2. X-Ray Assessment of Joints. The erosion of joints was scored according to Sharp/van der Heijde (SvH) [11]. The $\mathrm{SvH}$ scores were relatively stable during the study period (Figure 2(a)) with a mean score of 90.1 ( \pm 10.8 SEM, range $0-237)$ and 96.4 ( \pm 11.5 SEM, range $0-239)$, before and after 12 months with treatment, respectively. A few patients had a mild progression, and one patient had severe progression of joint erosion during the 12 months study period (Figure 2(b)).

\subsection{Serological Markers}

3.3.1. Cytokines. During the one-year study period, we monitored the levels of 12 common cytokines, representing a wide variety of immunological signalling functions (polarization). In general all serum cytokine levels were stable during the 
TABLE 1: Demographic data for the rheumatoid arthritis patient cohort.

\begin{tabular}{|c|c|c|c|c|c|c|c|c|}
\hline \multirow{2}{*}{ Patient number ${ }^{1}$} & \multirow{2}{*}{$\begin{array}{c}\text { Age of inclusion } \\
\text { (years) }\end{array}$} & \multirow{2}{*}{$\begin{array}{l}\text { RA duration }{ }^{2} \\
\text { (years) }\end{array}$} & \multirow{2}{*}{ Gender $(\mathrm{M}, \mathrm{F})^{\dagger}$} & \multicolumn{2}{|c|}{ Seropositive $^{3}$} & \multirow{2}{*}{ MTX dose $e^{4}$} & \multirow{2}{*}{$\begin{array}{l}\text { PRD dose } \\
(\mathrm{mg})\end{array}$} & \multirow{2}{*}{ IFX dose $^{6}$} \\
\hline & & & & Waaler & Anti-CCP & & & \\
\hline 101 & 60 & 5 & $\mathrm{~F}$ & + & + & 10 & 7,5 & 300 \\
\hline 102 & 45 & 14 & $\mathrm{~F}$ & + & + & 5 & 2,5 & 300 \\
\hline 106 & 50 & 22 & M & + & + & 7,5 & 0 & 300 \\
\hline 107 & 49 & 4 & $\mathrm{~F}$ & + & + & 7,5 & 5 & 300 \\
\hline 108 & 60 & 10 & $\mathrm{~F}$ & + & + & 10 & 2,5 & 200 \\
\hline 110 & 59 & 6 & $\mathrm{~F}$ & + & + & 7,5 & 0 & 200 \\
\hline 111 & 57 & 6 & $\mathrm{~F}$ & + & + & 15 & 2,5 & 200 \\
\hline 112 & 57 & 15 & $\mathrm{~F}$ & - & - & 5 & 7,5 & 400 \\
\hline 113 & 64 & 5 & $\mathrm{~F}$ & + & + & 10 & 0 & 200 \\
\hline 116 & 66 & 1 & $\mathrm{~F}$ & - & - & 10 & 7,5 & 300 \\
\hline 121 & 54 & 11 & $\mathrm{~F}$ & - & - & 2,5 & 7,5 & 200 \\
\hline 125 & 48 & 6 & M & + & + & 2,5 & 0 & 300 \\
\hline 126 & 35 & 8 & M & + & - & 15 & 10 & 300 \\
\hline 127 & 57 & 3 & $\mathrm{M}$ & + & + & 20 & 7,5 & 300 \\
\hline 130 & 68 & 11 & $\mathrm{M}$ & + & + & 15 & 0 & 300 \\
\hline 131 & 71 & 21 & $\mathrm{M}$ & + & + & 7,5 & 10 & 300 \\
\hline 132 & 56 & 3 & $\mathrm{~F}$ & + & + & 15 & 7,5 & 200 \\
\hline 133 & 30 & 11 & $\mathrm{~F}$ & + & + & 10 & 5 & 300 \\
\hline 134 & 54 & 18 & M & + & + & 10 & 5 & 300 \\
\hline 135 & 56 & 13 & $\mathrm{M}$ & - & - & 15 & 5 & 400 \\
\hline 136 & 52 & 7 & $\mathrm{~F}$ & + & + & 7,5 & 5 & 200 \\
\hline 137 & 21 & 3 & M & - & - & 15 & 5 & 300 \\
\hline 138 & 56 & 24 & $\mathrm{~F}$ & + & + & 20 & 5 & 300 \\
\hline 139 & 59 & 13 & M & + & + & 15 & 10 & 200 \\
\hline 201 & 58 & 7 & M & + & + & 15 & 5 & 300 \\
\hline 205 & 61 & 2 & $\mathrm{~F}$ & - & + & 15 & 0 & 200 \\
\hline 207 & 62 & 29 & $\mathrm{~F}$ & + & + & 12,5 & 2,5 & 200 \\
\hline 215 & 34 & 14 & $\mathrm{~F}$ & + & + & 15 & 10 & 200 \\
\hline 218 & 74 & 6 & F & + & + & 20 & 5 & 200 \\
\hline 223 & 44 & 15 & $\mathrm{~F}$ & + & + & 15 & 0 & 200 \\
\hline 233 & 59 & 2 & $\mathrm{~F}$ & + & + & 7,5 & 0 & 200 \\
\hline 302 & 64 & 5 & $\mathrm{~F}$ & - & - & 7,5 & 10 & 200 \\
\hline 303 & 74 & 21 & F & - & - & 7,5 & 5 & 300 \\
\hline 304 & 57 & 21 & F & - & + & 7,5 & 0 & 300 \\
\hline 305 & 57 & 26 & $\mathrm{M}$ & - & + & 15 & 0 & 300 \\
\hline 306 & 36 & 13 & M & + & + & 7,5 & 0 & 300 \\
\hline 307 & 56 & 18 & $\mathrm{M}$ & + & + & 20 & 0 & 300 \\
\hline 308 & 35 & 13 & F & - & + & 10 & 0 & 300 \\
\hline 309 & 43 & 21 & M & + & + & 12,5 & 5 & 200 \\
\hline
\end{tabular}

${ }^{1}$ Ref. no.: internal patient number, 1xx: Haugesund, 2xx: Bergen, and 3xx: Førde. ${ }^{2}$ Estimated duration of rheumatoid arthritis disease prior to infliximab treatment. ${ }^{3}$ Clinical data before treatment (baseline), +: seropositive, -: seronegative, ${ }^{4} \mathrm{MTX}$ : methotrexate, ${ }^{5} \mathrm{PRD}$ : prednisolone, ${ }^{6}$ IFX: infliximab, ${ }^{\dagger} \mathrm{F}$ : female, and M: male.

observation period (Figure 3). Interleukin-6 (Figure 3(e)) was the only cytokine that changed significantly ( $p$ values $<$ $0.05)$ during the course of anti-TNF treatment. The baseline level was $20 \mathrm{pg} / \mathrm{mL}$ and it fell to $4.5 \mathrm{pg} / \mathrm{mL}$ at 3 months after treatment initiation and remained at this level for the rest of the study period.
3.3.2. Matrix Metalloprotease (MMP). The serum levels of 5 MMPs were monitored, and none of the analysed MMPs changed significantly during the one-year study period (Figure 4). The average concentrations at baseline (with range) concentrations of the different MMPs were as follows: MMP-1 was $2781 \mathrm{pg} / \mathrm{mL}(145-18356 \mathrm{pg} / \mathrm{mL})$, 


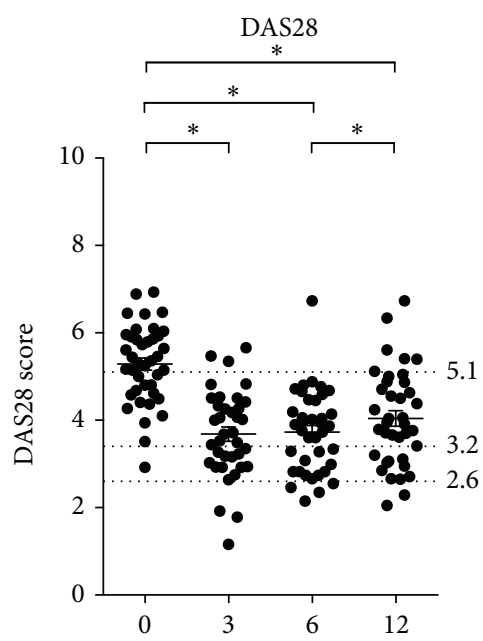

DAS28 $>5.1$ : high disease activity $3.2<$ DAS28 $\leq 5.1$ : moderate disease activity $2.6 \leq$ DAS2 283.2 : low disease activity DAS28 < 2.6: remission

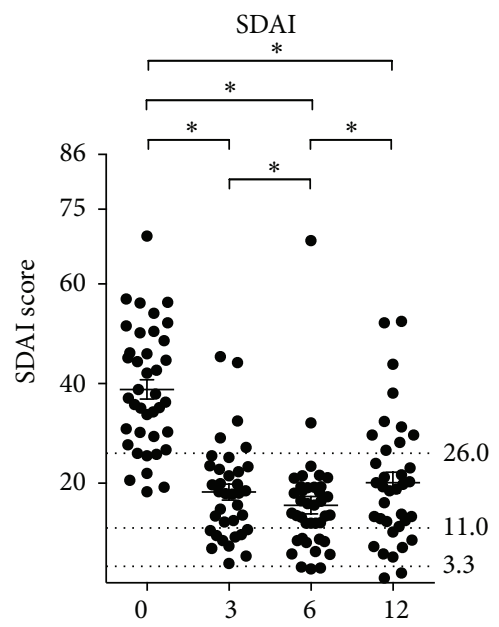

SDAI > 26.0: high disease activity $11.0<$ SDAI $\leq 26.0$ : moderate disease activity $3.3 \leq \mathrm{SDAI} \leq 11.0$ : low disease activity SDAI $<3.3$ : remission

(a)

(b)

FIGURE 1: DAS28 and SDAI scores. Scatter dot plots where each dot represents one individual patient. (a) SDAI and (b) DAS28 score distribution during the study period. The $y$-axes represent the disease activity score. The horizontal lines in the dot plot indicated the mean value. The dotted horizontal lines indicate borders between categories of disease activity: (i) high; (ii) moderate; (iii) low; and (iv) patients who are considered in remission. The $x$-axis is the four assessment time points indicated as $0,3,6$, and 12 months after treatment is initiated.

TABLE 2: Summary of the RA patient cohort data. Age and gender distribution and mean pharmacological doses.

\begin{tabular}{lc}
\hline Variable & \\
\hline Number of patients & 39 \\
Mean age \pm SD (range) & $53 \pm 12(21-74)$ \\
Gender ratio $\left(\mathrm{F}: \mathrm{M}^{1}\right)$ & $24: 15$ \\
RA duration $\pm \mathrm{SD}($ range) & $11 \pm 8(1-29)$ \\
RF-positive $(n / \%)$ & $28 / 72$ \\
Anti-CCP positive $(n / \%)$ & $31 / 80$ \\
Mean methotrexate dose $\pm \mathrm{SD}$ (range) & $11 \pm 5(2.5-20)$ \\
Mean infliximab dose $\pm \mathrm{SD}$ (range) & $264 \pm 58(200-400)$ \\
Mean prednisolone dose $\pm \mathrm{SD}$ (range) & $4 \pm 4(0-10)$ \\
\hline${ }^{1}$ Female to male ratio.
\end{tabular}

MMP-8 was $90.5 \mathrm{pg} / \mathrm{mL}(4.6-273.2 \mathrm{pg} / \mathrm{mL})$, MMP-13 was 98.9 $\mathrm{pg} / \mathrm{mL}$ (5.5-643.9 pg/mL), MMP-7 was $13.15 \mathrm{pg} / \mathrm{mL}$ (1.1-209.1 pg/mL), and MMP-12 was $620.7 \mathrm{pg} / \mathrm{mL}(20.6-$ $2402 \mathrm{pg} / \mathrm{mL})$.

3.3.3. Vascular Endothelial Growth Factor (VEGF). The mean concentration of VEGF-A at baseline was $1553 \mathrm{pg} / \mathrm{mL}$ with a range of 345.9 to $4476 \mathrm{pg} / \mathrm{mL}$, and after $12-$ month treatment the mean concentration of VEGF-A was $965.0 \mathrm{pg} / \mathrm{mL}$ with a range of 129.7 to $3128 \mathrm{pg} / \mathrm{mL}$. VEGF-A showed a significant decrease (Figure 5(a)) from baseline compared with months $3,6$, and 12 ( $p$ values $<0.05)$.

The mean concentration of VEGF-D at baseline was $39.4 \mathrm{pg} / \mathrm{mL}$ with a range of 1.0 to $255.5 \mathrm{pg} / \mathrm{mL}$, and 12 months after treatment the mean concentration of VEGF$\mathrm{D}$ was $42.64 \mathrm{pg} / \mathrm{mL}$ with a range of 1.0 to $339.5 \mathrm{pg} / \mathrm{mL}$. There was no significant change in VEGF-D when comparing baseline with 3,6 , or 12 months after treatment (Figure 5(b)). Additional results are presented in the supplementary material (in Supplementary Material available online at http://dx.doi.org/10.1155/2015/276815): distribution of ESR and CRP by gender and age (Supplementary Table 1) and frequencies of anti-CCP and rheumatoid factor (Supplementary Table 2); the clinical assessment of the RA patients (SWJ28, TEN28, ESR, CRP, MDGA-VAS, and PGA-VAS) during the study (Supplementary Figure 1); and the RA patients grouped by disease activity (Supplementary Figure 2) and the serum IgG-RF and anti-CCP titers during the study (Supplementary Figure 3).

\section{Discussion}

In this study, a cohort of 39 patients were selected from a prospective clinical open phase IV study which had a 3-year follow-up, where we focused on the first year of treatment. The aim was to examine the effect of infliximab treatment on disease status and progression in addition to several serological factors, which may be relevant serum markers for RA.

4.1. Decrease in Disease Activity. Already in 1999 Maini et al. showed clinical efficacy of infliximab treatment over 30 weeks in a phase III trial, where patients were treated with infliximab every 4 weeks concomitantly with methotrexate 


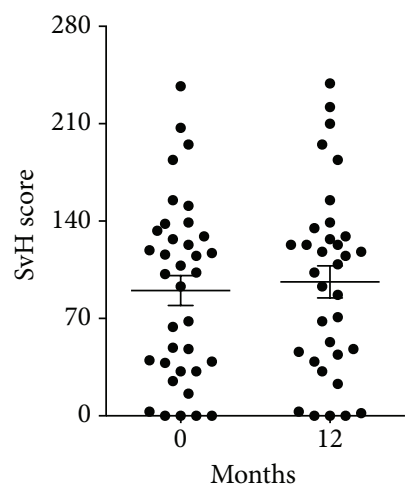

(a)

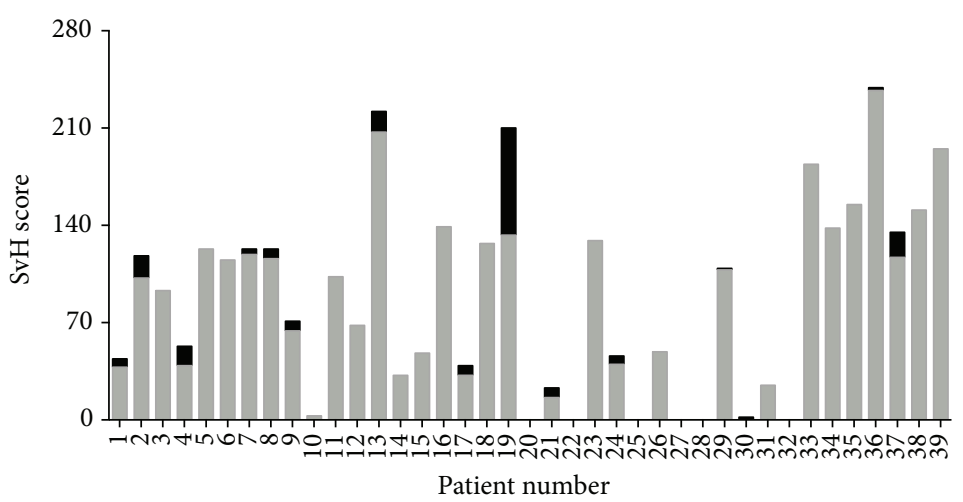

Baseline

Increase after 12 months

FIGURE 2: Progression of joint erosion. Erosions in the affected joints of the RA cohort, where the $y$-axes represent the Sharp/van der Heijde scores; (a) the $x$-axis illustrates the two assessment time points indicated as 0 and 12 months after treatment in a paired $t$-test; (b) the $x$-axis represents the patients' individual progression in joint erosion in a histogram, where the grey bars illustrate baseline joint erosions and the black bars illustrate additional joint erosions after 12 months.

[12]. Our patients were in general in an advanced stage of disease with severe joint affection and tissue destruction when considering the clinical data (Figure 1). Collectively, the patient cohort disease activity and inflammation status decreased significantly, indicating that our patients benefited from the infliximab treatment. Several studies have been performed in an effort to ascertain anti-TNF- $\alpha$ treatment efficacy compared with DMARDs alone, and many report a positive effect [13-15]. The Sharp/van Der Heijde scores from the high-resolution X-ray imaging support that there was no significant change in joint damage collectively in the cohort (Figure 2), comparing baseline with 12 months after initiation of treatment. Only one patient showed extensive progression in joint damage, but this patient had severe joint damage already before entering the study.

\subsection{Serum Markers}

4.2.1. Cytokines. Cytokines are important signaling mole-cules facilitating communication between cells of the immune system and useful as markers for ongoing immune reactions. We tested the serum level of a range of cytokines (IL-1 $\beta$, IL-2, IL-4, IL-5, IL-6, IL-10, IL-12p70, IL-17A, IL-18, IFN- $\gamma$, TNF$\alpha$, and TNF- $\beta$ ); these cytokines represent a wide range of functional immune messengers.

Only IL-6 changed during the course of infliximab treatment, and it decreased significantly (Figure 3(e)). It has been suggested that IL- $1 \beta$ modifies IL- 6 induced acute phase proteins in the liver by upregulation of CRP production [16]. This relationship between IL-6 and CRP might be one explanation for the apparent correlation between CRP and IL-6 reduction. Additionally, it has recently been proposed that baseline serum levels of IL- 6 , but not TNF- $\alpha$, are a possible serum marker for disease activity in early RA [17].
Serum levels of TNF- $\alpha$ in healthy control sera have been reported to be lower than $6 \mathrm{pg} / \mathrm{mL}$ in a case study of a patient with large granular lymphocyte syndrome (LGL) [18]. Also, in a study of healthy subjects the mean TNF- $\alpha$ serum levels were reported to be between 30 and $40 \mathrm{pg} / \mathrm{mL}$ depending on age [19]. Our patients had an average of $1.8 \mathrm{pg} / \mathrm{mL}$ TNF- $\alpha$ at the start of the study, and the serum levels rose slightly to $7.3 \mathrm{pg} / \mathrm{mL}$ after 12 months, but this increase was not statistically significant.

4.2.2. Matrix Metalloproteases. MMPs belong to a group of zinc-dependant endopeptidases involved in catalytic breakdown of extracellular matrix (ECM) proteins. Additionally, these enzymes serve important functions in tissue formation and remodelling, growth, wound healing, and maintenance. MMPs may contribute to tissue damage and break down if not properly regulated, and this is partly controlled by MMP inhibitors called Tissue Inhibitors of Metalloproteases (TIMPs).

We have tested MMPs that target collagen (MMP-1, MMP-8, and MMP-13), gelatin, fibronectin, and proteoglycans (MMP-7) and elastin and basement membrane components (MMP-12) [20]. We did not observe any change in MMPs levels, and they remained low during the study. This finding supports that inflammatory responses or joint erosion is not significantly increased during the study period.

Serum levels of MMP-3 in particular have previously been found to correlate with disease activity in RA and MMP9 in systemic lupus erythematosus (SLE), which is another rheumatic disease [21, 22]. MMP-3 and MMP-9 have also been found to contribute to inflammatory conditions in the skin [20]. It has also been reported that infliximab reduces MMP-3 and MMP-9 levels in peripheral blood monocytes in patients with active RA [23]. Since we did not observe any 


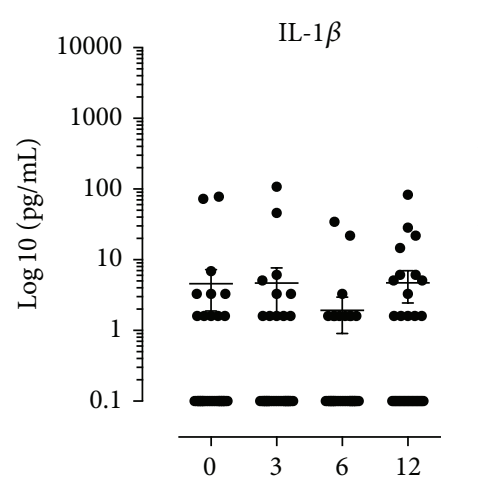

(a)

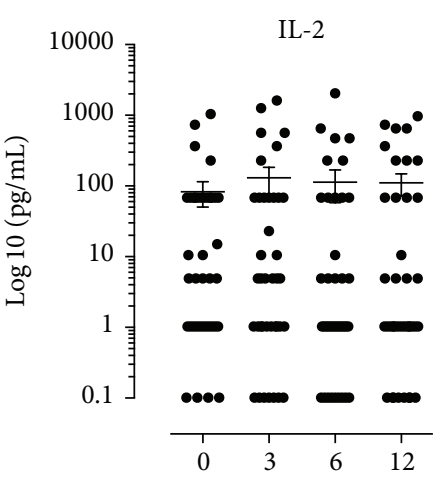

(b)

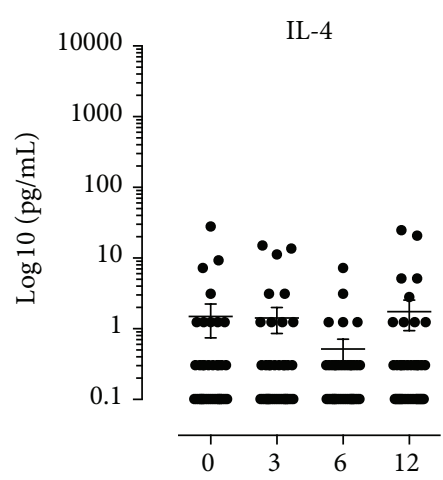

(c)

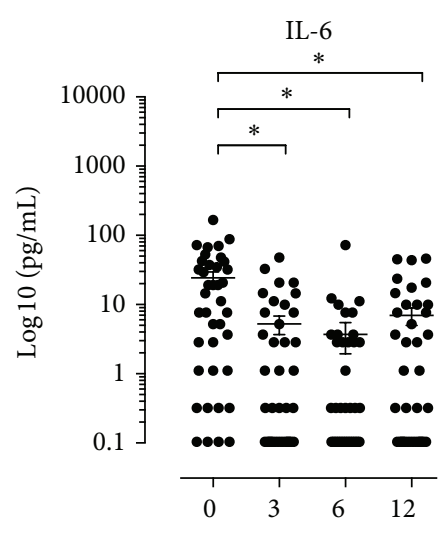

(e)

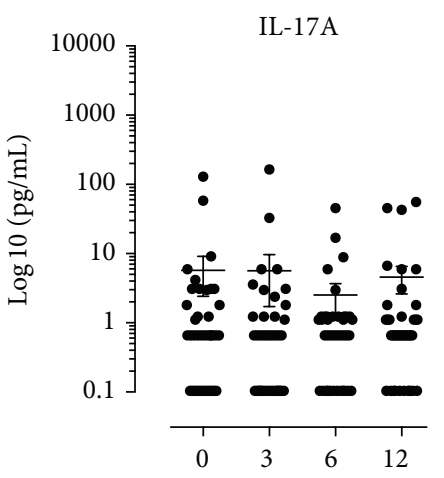

(h)

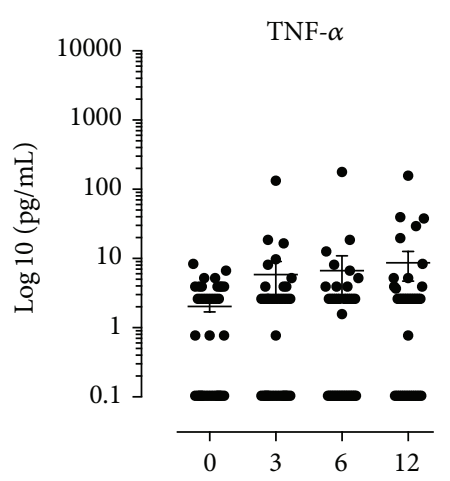

(k)

\section{IL-10}

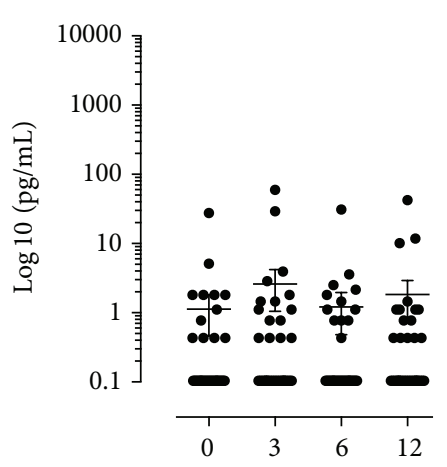

(f)

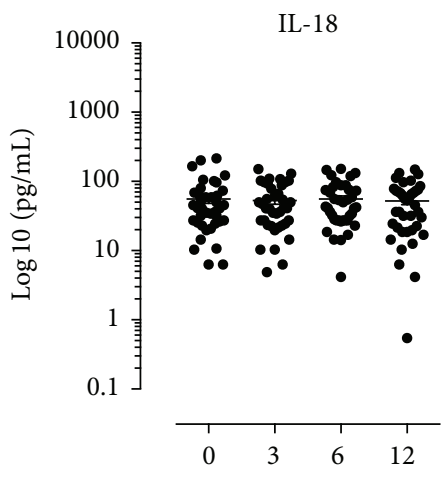

(i)

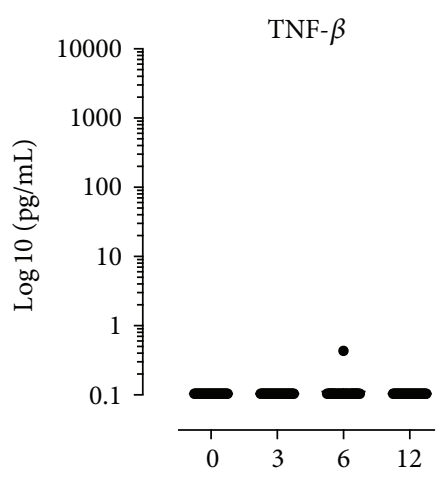

(1)

FIGURE 3: The serum cytokine levels in the rheumatoid arthritis patients. (a) to (l) are scatter dot plots of serum cytokine levels, where each dot represents one individual patient. The $x$-axes illustrate the four assessment months after infliximab treatment is initiated. The concentrations in serum of their respective cytokine in picograms per millilitre $(\mathrm{pg} / \mathrm{mL})$ on a $\log 10$ scale. The horizontal lines in the dot plot illustrate the mean, and the vertical error lines represent \pm standard error mean (SEM) in a paired $t$-test. 


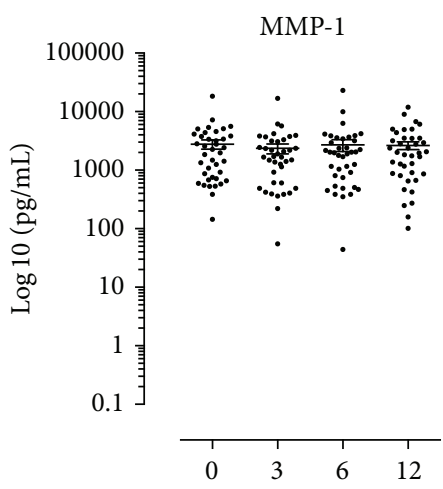

(a)

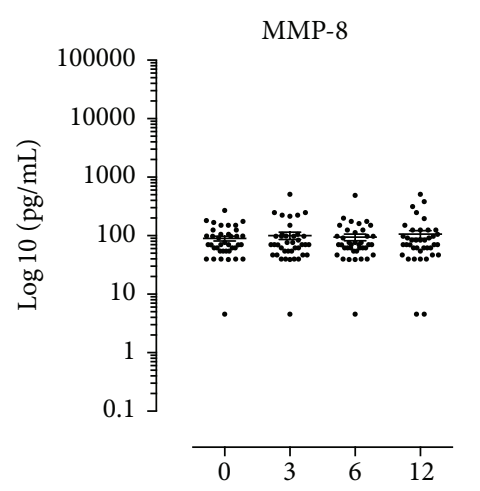

(b)

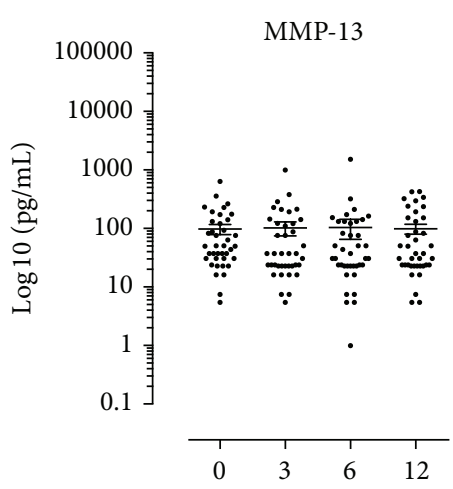

(c)

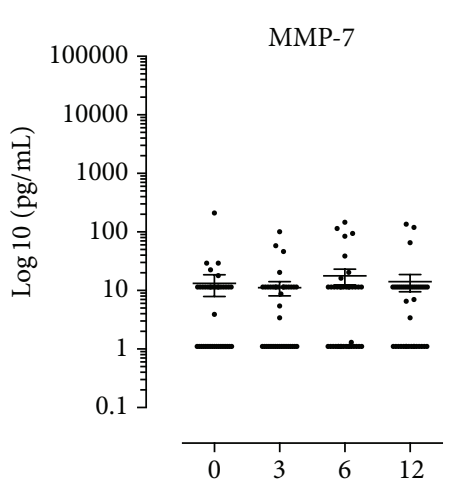

(d)

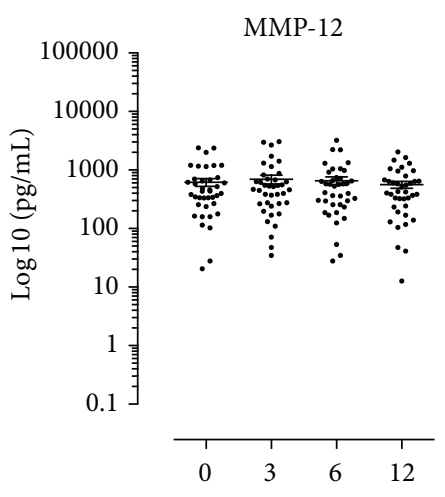

(e)

Figure 4: Matrix metalloprotease levels in serum from the RA patient cohort. (a) to (e) are scatter dot plots, where each dot represents one individual patient. The $x$-axes illustrate the four assessment months after infliximab treatment is initiated. The $y$-axes represent the concentrations in serum of their respective MMP in picograms per millilitre (pg/mL) on a $\log 10$ scale. The horizontal lines in the dot plot illustrate the mean, and the vertical error lines represent \pm standard error mean (SEM) in a paired $t$-test.

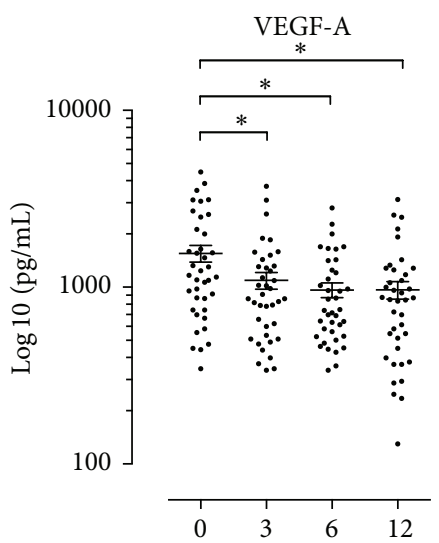

(a)

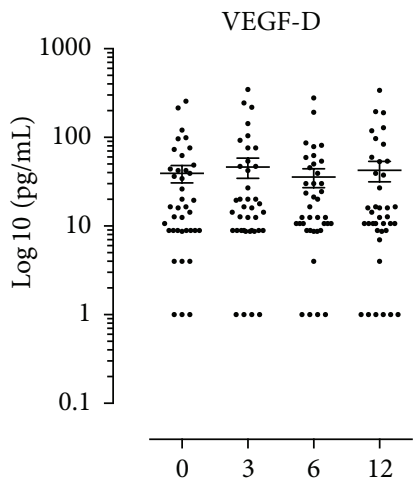

(b)

Figure 5: The VEGF-A and VEGF-D serum concentrations. The $x$-axes illustrate the four assessment months after infliximab treatment is initiated. The $y$-axes represent the concentrations in serum of their respective VEGF in picograms per millilitre (pg/mL) on a log $10 \mathrm{scale}$. The horizontal lines in the dot plot illustrate the mean, and the vertical error lines represent \pm standard error mean (SEM) in a paired $t$-test.

significant disease progression in this study, these MMPs may also be stable in our cohort.

4.2.3. Vascular Endothelial Growth Factors. We observed a weak decrease in serum concentration of VEGF-A throughout the study period (Figure 5(a)). Increased VEGF levels have been shown to induce TNF and IL-6 production $[24,25]$, which may promote inflammatory reactions. There may be a mutual and perhaps synergistic relationship between VEGFs and TNF/IL-6, as we observe a decrease 
in both IL-6 and VEGF-A in patients undergoing anti-TNF treatment, and with concurrent stable levels of joint destruction.

Vascular endothelial growth factors (VEGFs) consist of a family of cytokines and growth factors and are generally produced by cells in tissue that is oxygen depleted [26]. They mainly target endothelial cells via receptors (VEGF-R) and induce angiogenesis, that is, formation of new blood vessels. Many types of cells are capable of VEGF production and secretion, and they may also stimulate different cells, not only endothelial cells.

By inducing angiogenesis, VEGF is an important factor in facilitating cell adhesion and inflammation by promoting cell adhesion molecules and, in turn, in the recruitment of mononuclear cells like macrophages to target tissues [27]. VEGFs have been associated with development of joint destruction in RA, and it has been postulated that the destruction is mediated through the expansion of synovial vasculature [28-31].

Additionally, fibroblasts are reportedly stimulated by cytokines such as IL-1 and TNF- $\alpha$ to produce VEGF [25], which in turn can lead to increased proliferation of endothelial cells and vascularisation. VEGF has previously been associated with disease activity in RA and increased vascularisation may lead to increased leukocyte migration and mediate inflammation via this mechanism. VEGF has also been reported to correlate with erythrocyte sedimentation rate (ESR) and to contribute to the production of TNF and IL-6.

In conclusion, based on the various assessments and laboratory measurements performed in the clinic and the laboratory, we have shed some light on underlying features of the serology in rheumatoid arthritis. The study population showed a significant decrease in disease activity, indicating that anti-TNF treatment is efficacious despite long duration of RA. We observed no change in serum concentrations of many proinflammatory cytokines, TNF- $\alpha$, and matrix metalloproteases during treatment. Nevertheless, we found statistically significant changes in serum levels of IL-6 and VEGF-A varying with disease activity. The clinical significance of this finding has to be further elucidated and confirmed in larger clinical trials before being utilized in a clinical setting.

\section{Conflict of Interests}

The authors declare that there is no conflict of interests regarding the publication of this paper.

\section{Acknowledgments}

The authors want to thank Kjerstin Jakobsen and Marianne Eidsheim for excellent technical assistance. Special thanks go to the specialists in rheumatology for providing the clinical data and to the laboratory staff for collecting samples. Thanks to Rebecca J. Cox for comments and suggestions preparing this paper. This study was funded by the Broegelmann Foundation, Center for Pharmacy (UiB), and Gades Legat.

\section{References}

[1] P. S. Gulko and R. J. Winchester, "Rheumatoid arthitis," in Samter's Immunologic Diseases, K. F. Austen, M. M. Frank, J. P. Atkinson, and H. Cantor, Eds., chapter 37, pp. 427-463, Lippincott Williams \& Wilkins, Philadelphia, Pa, USA, 6th edition, 2001.

[2] A. L. M. A. Jansen, I. E. van der Horst-Bruinsma, D. van Schaardenburg, R. J. van de Stadt, M. H. M. T. de Koning, and B. A. C. Dijkmans, "Rheumatoid factor and antibodies to cyclic citrullinated peptide differentiate rheumatoid arthritis from undifferentiated polyarthritis in patients with early arthritis," Journal of Rheumatology, vol. 29, no. 10, pp. 2074-2076, 2002.

[3] A. Kastbom, G. Strandberg, A. Lindroos, and T. Skogh, "AntiCCP antibody test predicts the disease course during 3 years in early rheumatoid arthritis (the Swedish TIRA project)," Annals of the Rheumatic Diseases, vol. 63, no. 9, pp. 1085-1089, 2004.

[4] I. K. Gao, A. Haas-Wöhrle, K. G. Mueller, H.-M. Lorenz, and C. Fiehn, "Determination of anti-CCP antibodies in patients with suspected rheumatoid arthritis: does it help to predict the diagnosis before referral to a rheumatologist?" Annals of the Rheumatic Diseases, vol. 64, no. 10, pp. 1516-1517, 2005.

[5] M. L. L. Prevoo, M. A. van'T Hof, H. H. Kuper, M. A. van Leeuwen, L. B. A. van De Putte, and P. L. C. M. van Riel, "Modified disease activity scores that include twenty-eight-joint counts: development and validation in a prospective longitudinal study of patients with rheumatoid arthritis," Arthritis \& Rheumatism, vol. 38, no. 1, pp. 44-48, 1995.

[6] J. S. Smolen, F. C. Breedveld, M. H. Schiff et al., "A simplified disease activity index for rheumatoid arthritis for use in clinical practice," Rheumatology, vol. 42, no. 2, pp. 244-257, 2003.

[7] Y. Yazici and I. Simsek, "Tools for monitoring remission in rheumatoid arthritis: any will do, let's just pick one and start measuring," Arthritis Research and Therapy, vol. 15, no. 1, article 104, 2013.

[8] D. Aletaha, T. Neogi, A. J. Silman et al., "2010 Rheumatoid arthritis classification criteria: an American College of Rheumatology/European League Against Rheumatism collaborative initiative," Arthritis and Rheumatism, vol. 62, no. 9, pp. 25692581, 2010.

[9] F. C. Arnett, S. M. Edworthy, D. A. Bloch et al., "The American Rheumatism Association 1987 revised criteria for the classification of rheumatoid arthritis," Arthritis and Rheumatism, vol. 31, no. 3, pp. 315-324, 1988.

[10] A. G. Kvalvik, L. Lefsaker, S. Dyvik, and J. G. Brun, "Anti-tumor necrosis factor-alpha therapy in the ordinary clinical setting: three-year effectiveness in patients with rheumatoid arthritis," Joint Bone Spine, vol. 74, no. 6, pp. 606-611, 2007.

[11] D. M. F. M. van der Heijde, "Radiographic imaging: the 'gold standard' for assessment of disease progression in rheumatoid arthritis," Rheumatology, vol. 39, supplement 1, pp. 9-16, 2000.

[12] R. Maini, E. W. St Clair, F. Breedveld et al., "Infliximab (chimeric anti-tumour necrosis factor $\alpha$ monoclonal antibody) versus placebo in rheumatoid arthritis patients receiving concomitant methotrexate: a randomised phase III trial," The Lancet, vol. 354, no. 9194, pp. 1932-1939, 1999.

[13] H. T. Ozer and Z. Ozbalkan, "Clinical efficacy of TNF- $\alpha$ inhibitors: an update," International Journal of Clinical Rheumatology, vol. 5, no. 1, pp. 101-115, 2010.

[14] R. N. Maini, F. C. Breedveld, J. R. Kalden et al., "Therapeutic efficacy of multiple intravenous infusions of anti-tumor necrosis factor alpha monoclonal antibody combined with low-dose 
weekly methotrexate in rheumatoid arthritis," Arthritis and Rheumatism, vol. 41, no. 9, pp. 1552-1563, 1998.

[15] M. Feldmann and R. N. Maini, "Anti-TNF therapy, from rationale to standard of care: what lessons has it taught us?" The Journal of Immunology, vol. 185, no. 2, pp. 791-794, 2010.

[16] J. G. Bode, U. Albrecht, D. Häussinger, P. C. Heinrich, and F. Schaper, "Hepatic acute phase proteins-regulation by IL-6and IL-1-type cytokines involving STAT3 and its crosstalk with NF- $\kappa$ B-dependent signaling," European Journal of Cell Biology, vol. 91, no. 6-7, pp. 496-505, 2012.

[17] K. Shimamoto, T. Ito, Y. Ozaki et al., "Serum interleukin 6 before and after therapy with tocilizumab is a principal biomarker in patients with rheumatoid arthritis," Journal of Rheumatology, vol. 40, no. 7, pp. 1074-1081, 2013.

[18] M. Ozaki, Y. Kawabe, H. Nakamura et al., "Elevated serum cytokine levels in a rheumatoid arthritis patient with large granular lymphocyte syndrome," Rheumatology, vol. 40, no. 5, pp. 592-593, 2001.

[19] G. Kleiner, A. Marcuzzi, V. Zanin, L. Monasta, and G. Zauli, "Cytokine levels in the serum of healthy subjects," Mediators of Inflammation, vol. 2013, Article ID 434010, 6 pages, 2013.

[20] A. Page-McCaw, A. J. Ewald, and Z. Werb, "Matrix metalloproteinases and the regulation of tissue remodelling," Nature Reviews Molecular Cell Biology, vol. 8, no. 3, pp. 221-233, 2007.

[21] R. Carrasco and A. Barton, "Biomarkers of outcome in rheumatoid arthritis," Rheumatology Reports, vol. 2, no. 1, pp. 26-38, 2010.

[22] A. Ban, M. Inaba, Y. Furumitsu et al., "Time-course of health status in patients with rheumatoid arthritis during the first year of treatment with infliximab," Biomedicine and Pharmacother$a p y$, vol. 64, no. 2, pp. 107-112, 2010.

[23] J. Huang, B. Xie, Q. Li et al., "Infliximab reduces CD147, MMP3, and MMP-9 expression in peripheral blood monocytes in patients with active rheumatoid arthritis," European Journal of Pharmacology, vol. 698, no. 1-3, pp. 429-434, 2013.

[24] S.-A. Yoo, S.-K. Kwok, and W.-U. Kim, "Proinflammatory role of vascular endothelial growth factor in the pathogenesis of rheumatoid arthritis: prospects for therapeutic intervention," Mediators of Inflammation, vol. 2008, Article ID 129873, 6 pages, 2008.

[25] Z. Szekanecz and A. E. Koch, "VEGF as an activity marker in rheumatoid arthritis," International Journal of Clinical Rheumatology, vol. 69, pp. 1172-1178, 2010.

[26] S. Konisti, S. Kiriakidis, and E. M. Paleolog, "Hypoxia-a key regulator of angiogenesis and inflammation in rheumatoid arthritis," Nature Reviews Rheumatology, vol. 8, no. 3, pp. 153$162,2012$.

[27] G. Murdaca, F. Spanò, M. Miglino, and F. Puppo, "Effects of TNF- $\alpha$ inhibitors upon the mechanisms of action of VEGF," Immunotherapy, vol. 5, no. 2, pp. 113-115, 2013.

[28] S. Ballara, P. C. Taylor, P. Reusch et al., "Raised serum vascular endothelial growth factor levels are associated with destructive change in inflammatory arthritis," Arthritis and Rheumatism, vol. 44, no. 9, pp. 2055-2064, 2001.

[29] L. Ozgonenel, E. Cetin, S. Tutun, P. Tonbaklar, H. Aral, and G. Guvenen, "The relation of serum vascular endothelial growth factor level with disease duration and activity in patients with rheumatoid arthritis," Clinical Rheumatology, vol. 29, no. 5, pp. 473-477, 2010.

[30] H. R. Kim, K. W. Kim, B. M. Kim, M. L. Cho, and S. H. Lee, "The effect of vascular endothelial growth factor on osteoclastogenesis in rheumatoid arthritis," PLoS ONE, vol. 10, no. 4, Article ID e0124909, 2015.

[31] S. Kelly, M. Bombardieri, F. Humby et al., "Angiogenic gene expression and vascular density are reflected in ultrasonographic features of synovitis in early rheumatoid arthritis: an observational study," Arthritis Research \& Therapy, vol. 17, article $18,2015$. 


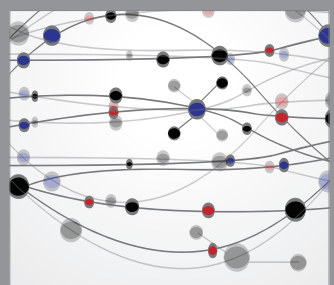

The Scientific World Journal
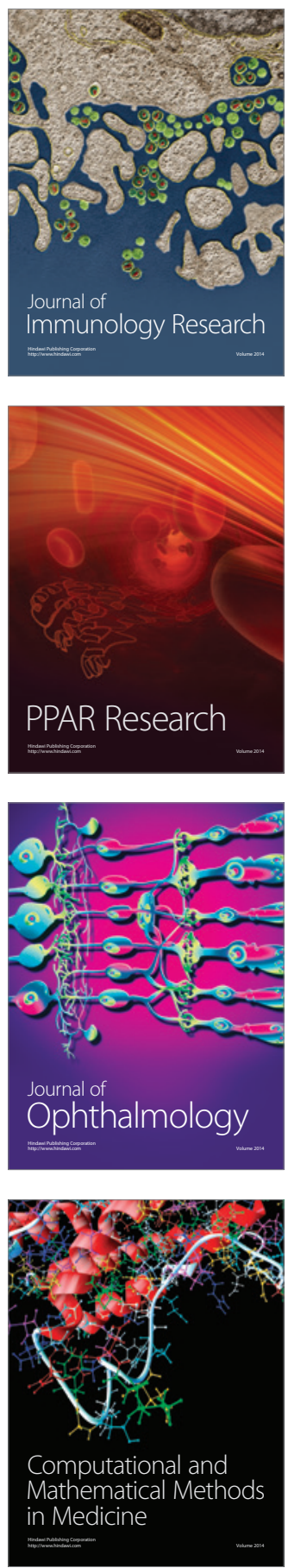

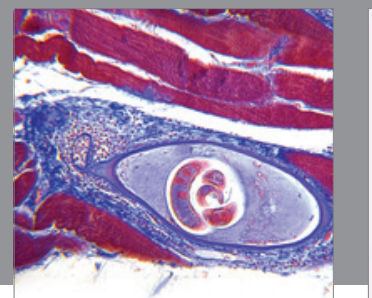

Gastroenterology

Research and Practice
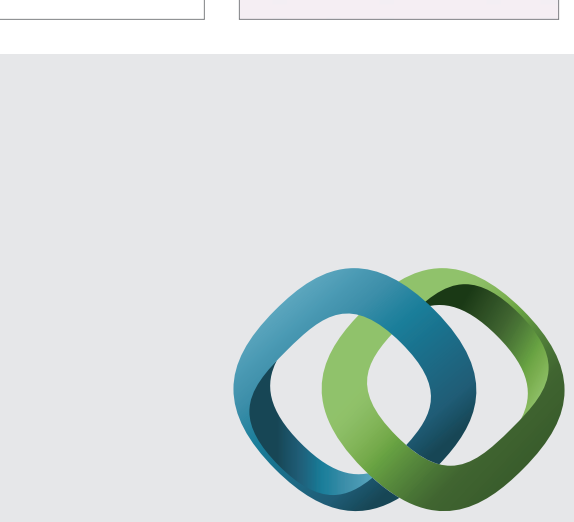

\section{Hindawi}

Submit your manuscripts at

http://www.hindawi.com
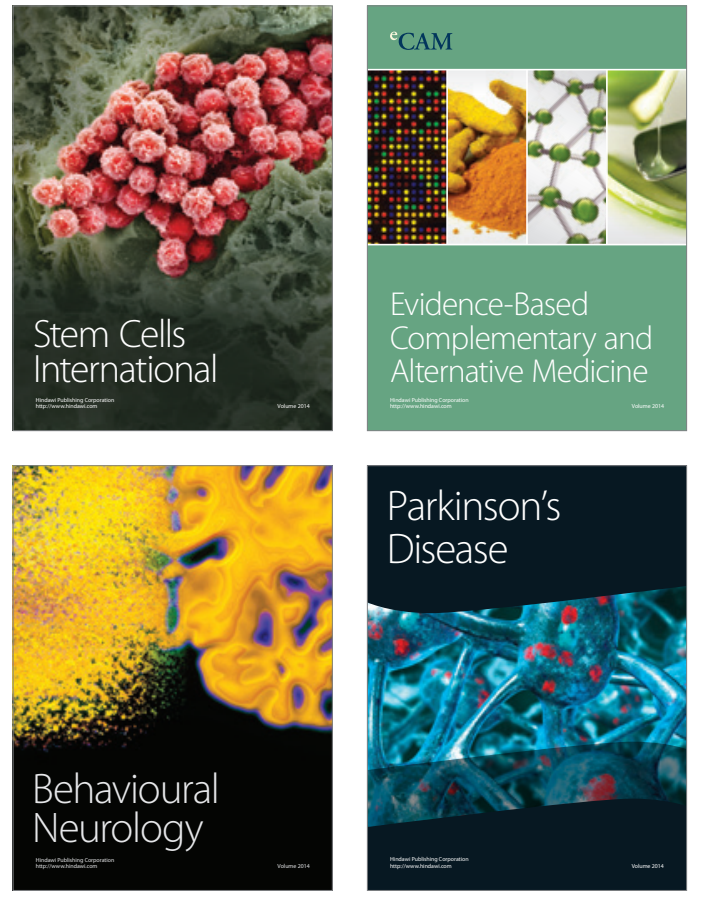
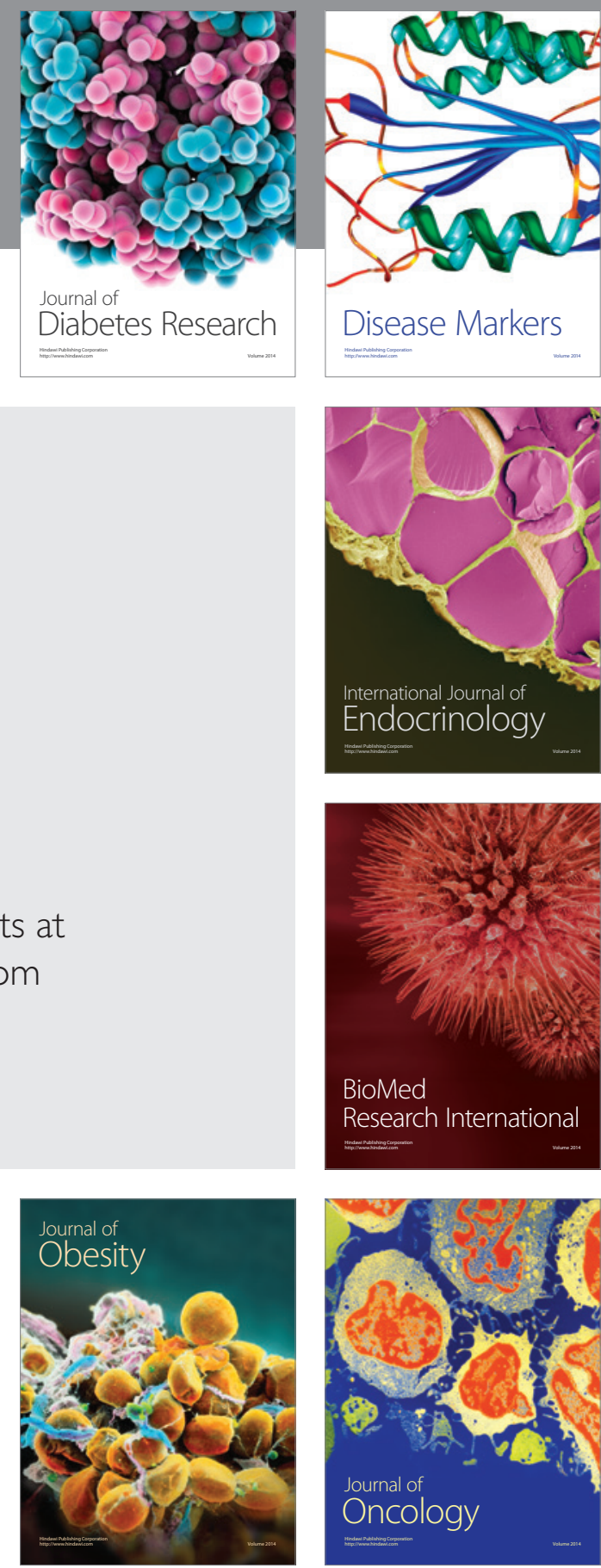

Disease Markers
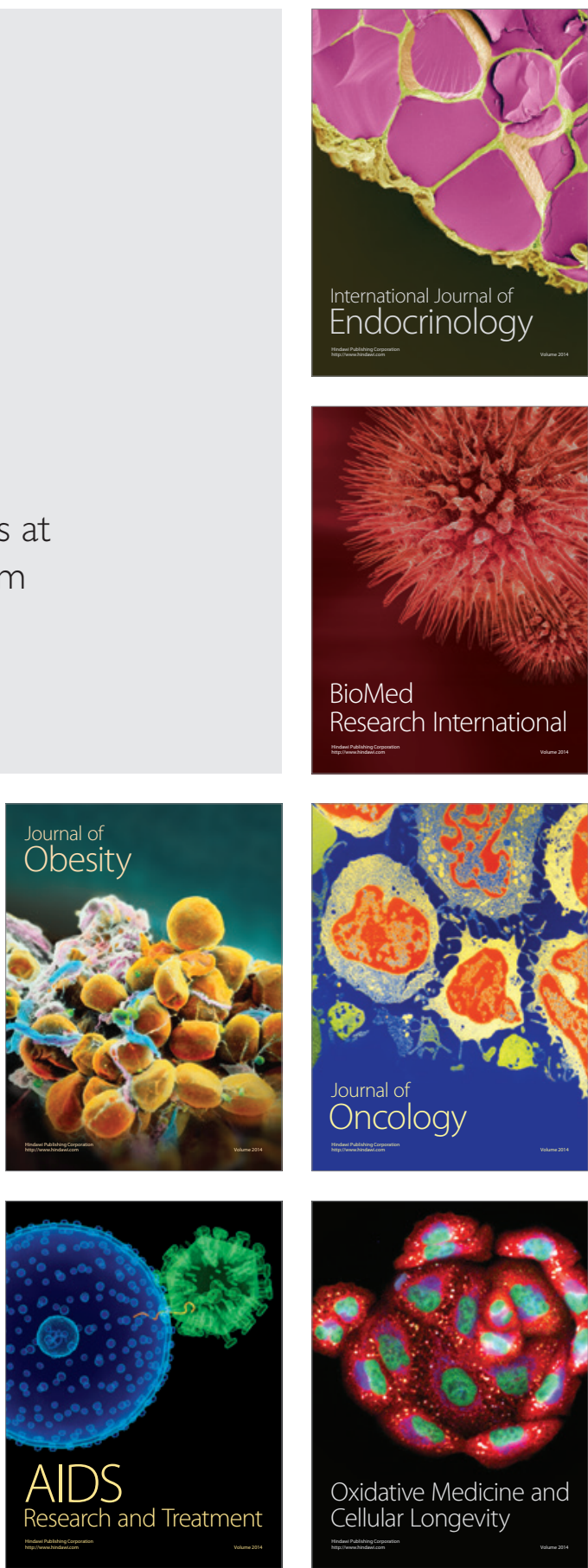